\title{
Ogawa Growth Medium
}

National Cancer Institute

\section{Source}

National Cancer Institute. Ogawa Growth Medium. NCI Thesaurus. Code C85508.

A growth medium that is especially used for the cultivation of mycobacterium consisting of LJ Medium without the asparagine. 